\title{
Eficiência dos Ésteres de Fitoesteróis Alimentares na Redução dos Lípides Plasmáticos em Hipercolesterolêmicos Moderados
}

\author{
Ana M. P. Lottenberg, Valéria S. Nunes, Edna R. Nakandakare, Mônica Neves, Márcia Bernik, \\ José E. Santos, Eder C. R. Quintão \\ São Paulo, SP
}

Objetivo - Avaliar o efeito dos ésteres de fitoesteróis (FE) da dieta, em função dos diferentes genótipos de apo E, sobre os lípides plasmáticos em indivíduos moderadamente hipercolesterolêmicos.

Métodos - Pacientes moderadamente hipercolesterolêmicos (20 a 60 anos de idade; 50 mulheres e 10 homens), mantidos em dieta habitual, receberam margarina (20g/dia) enriquecida com fitoesteróis $(2,8 \mathrm{~g} /$ dia $=1,68 \mathrm{~g}$ de FE), ou margarina normal (placebo), por dois períodos de quatro semanas cada, em estudo duplo cego e cruzado.

Resultados - Fitoesteróis reduziram significativamente o colesterol total e o LDL-colesterol, respectivamente, em $10 \%$ e 12\% quando comparado à fase basal de admissão ao programa, e $6 \%$ e $8 \%$ quando comparado à fase placebo. $O$ HDL-colesterol e a trigliceridemia não se modificaram.

Conclusão - Ésteres defitoesteróis da dieta reduziram a colesterolemia e a redução do LDL-colesterol foi mais pronunciada nos individuos que apresentavam valores maiores de LDL-colesterol, quando admitidos no estudo.

Em relação ao sinótipo de apo E não houve diferença significativa entre apo $E 3 / 3$ e apo $E 3 / 4$.

Palavras-chave: ésteres de fitoesteróis, colesterol, LDLcolesterol

\section{Plant sterol ester Efficiency on the Plasma Lipid Reduction in Moderate Hipercholesterolemic Subjects}

Objective - This study aimed at relating the pattern of response to dietary plant sterol ester (PSE) treatment of plasma lipids concentrations and apo E polymorphisms.

Methods - Patients (20-60y old: 50 women; 10 men) with primary moderate hypercholesterolemia were fed margarine $(20 \mathrm{~g} / \mathrm{d})$, received no treatment (placebo), and were fed PSE (2.8g/d = 1.68 phytosterols $)$, during 3 periods of 4 weeks each, in a crossover, double-blind study. DNA was extracted from white blood cells for the apo E polymorphisms.

Results - PSE treatment significantly lowered TC and $L D L-C 10 \%$ and $12 \%$, respectively, in relation to the baseline, and $6 \%$ and $8 \%$ in relation to the placebo phase, but $H D L-C$ and TG levels were not modified. In regard to the apo E genotyping, no significant difference occurred between apo $E 3 / 3$ and apo $E 3 / 4$.

Conclusion - Dietary plant sterol ester (PSE) treatmentreduced cholesterolemia, and the reduction of $L D L-C$ in absolute values was more pronounced when the initial LDL - C concentration were elevated.

Keywords: plant sterol ester, cholesterol, LDL-colesterol, apo E

\footnotetext{
Laboratório de Lípides do Hospital das Clínicas da FMUSP (LIM-10) e Faculdade de Medicina de Ribeirão Preto da USP

Correspondência: Eder C. R. Quintão - Laboratório de Lípides da FMUSP Av. Dr. Arnaldo, 455 - $3^{\circ}$ - S/3317 - 01246-000 - São Paulo, SP - E-mail: lipideq@usp.br Recebido para publicação em 27/6/01 Aceito em 4/2/02
} 
Estudos clínicos e experimentais demonstraram que a adição de fitoesteróis na dieta reduz os níveis plasmáticos de colesterol e de LDL-colesterol ${ }^{1}$. Em humanos, há necessidade de no mínimo, $3 \mathrm{~g} /$ dia de fitoesteróis para redução da colesterolemia, embora as concentrações de HDL-colesterol e triglicérides não se alterem ${ }^{2-4}$. Esses resultados levaram ao enriquecimento de margarinas comercialmente disponíveis, com ésteres de fitoesteróis ou fitoestanóis, que são os fitoesteróis reduzidos ${ }^{5-8}$.

Durante os últimos 10 anos, várias publicações examinaram as interações gene dieta, nas quais foi estudada a resposta dos lípides plasmáticos, em função de alterações no teor de gordura e de colesterol da dieta ${ }^{9-12}$.

As lipoproteínas do plasma contêm diversas apolipoproteínas, como as apolipoproteína $\mathrm{E}$ (apoE), que controlam o seu metabolismo ${ }^{13,14}$. Emhumanos, a apoEé um componente estrutural de quilomícrons e remanescentes de VLDL, os quais regulam a ligação e a captação celular de LDL. Assim, mutações nas apoE foram descritas como responsáveis pela ocorrência de várias formas de dislipidemias. Há três genes principais de apoE em toda população humana: E2, E3, E4. Assim, estes genes caracterizam fenótipos de apoE, sendo três homozigóti$\cos (\mathrm{E} 2 / \mathrm{E} 2, \mathrm{E} 3 / \mathrm{E} 3, \mathrm{E} 4 / \mathrm{E} 4)$ e três heterozigóticos(E2/E3, E2/E4, $\mathrm{E} 3 / \mathrm{E} 4)$. As concentrações de colesterol total e triglicérides no plasma, representadas principalmente pelas lipoproteínas de baixa densidade(LDL,VLDL), dependem do genótipo apo E. O genótipo E2 eleva triglicérides e reduz o colesterol total, enquanto E4 reduz triglicérides e eleva o colesterol total, ao mesmo tempo que E3 desempenha papel intermediário entre as outras duas apoE. Sabe-se que os indivíduos E3/4, absorvem maior quantidade de colesterol da dieta ${ }^{15,16}$. No entanto, a resposta da colesterolemia com o uso de fitoesteróis em função dos diferentes fenótipos de apoE não está ainda totalmente elucidada na literatura.

Todas as investigações sobre os efeitos dos fitoesteróis foram conduzidas no exterior onde os hábitos alimentares diferem da nossa população e com níveis de lípides plasmáticos mais elevados do que os encontrados na população brasileira ${ }^{17}$. Por exemplo, margarinas modificadas foram estudadas entre os finlandeses que, além de ingerirem quantidade maior de margarina do que os brasileiros, também apresentam média de colesterolemia mais elevada ${ }^{17}$. Desta forma, este trabalho objetivou medir a resposta dos lípides plasmáticos, aos ésteres de fitoesteróis em função dos diferentes fenótipos de apo E em uma situação de ingestão de margarina acima da habitual de nossa população e em indivíduos com moderada elevação da colesterolemia. Estes pacientes constituem a maior parte dos hipercolesterolêmicos que, portanto, com maior freqüência, fazem uso de fármacos redutores da colesterolemia e poderiam beneficiar-se com modificações alimentares.

\section{Métodos}

Foram estudados 60 indivíduos, de ambos sexos, portadores de hipercolesterolemia primária moderada, ou seja, com colesterol plasmático entre $240 \mathrm{mg} / \mathrm{dL}$ e $300 \mathrm{mg} / \mathrm{dL}$, normotrigliceridêmicos (triglicérides $<250 \mathrm{mg} / \mathrm{dL}$ ) e com LDLcolesterol $>130 \mathrm{mg} / \mathrm{dL}$. A vantagem de se trabalhar com essa faixa é, exatamente, por ser a forma mais comum de elevação da colesterolemia populacional. Foram excluídos do estudo indivíduos com índice de massa corpórea acima de 30 e portadores de diabetes, hipotireoidismo, doença hepática ou renal, ou em uso de medicação que interferisse nos lípides do plasma.

O estudo foi duplo-cego cruzado, com seleção de participantes no Ambulatório de Dislipidemias do Hospital das Clínicas da FMUSP e Faculdade de Medicina Ribeirão Preto, com duração de quatro semanas em cada dieta experimental, portanto oito semanas ao todo. O projeto foi aprovado pela comissão de ética de ambas as instituições e todos os pacientes assinaram termo de consentimento para a realização do estudo. A seleção dos casos foi feita baseada em três medidas de lípides plasmáticos, com intervalo de uma semana a ummês.

Os pacientes compareceram semanalmente ao hospital para controle de peso corporal, coleta de sangue e também para receber os potes contendo a margarina. Os potes continham $20 \mathrm{~g}$ de margarina (dose diária) e cada paciente recebeu quantidade suficiente para uma semana, rotulados como "margarina A" ou "margarina B". Os pacientes foram mantidos em sua dieta habitual e utilizaram margarina placebo ou enriquecida com FE por quatro semanas cada. Metade dos pacientes iniciou com margarina rotulada como "margarina A" e a outra metade com "margarina B". Após quatro semanas houve inversão de A para B e de B para A, respectivamente, portanto sem wash out intermediário. Os pacientes foram orientados a ingerir toda a quantidade de margarina, que poderia ser utilizada em legumes, pão ou bolacha, durante três refeições diárias, totalizando aproximadamente $3 \mathrm{~g}$ fitoesteróis por dia, ou seja, 1,68g de fitoesterol, sendo aproximadamente $45 \%$ de b-sitosterol e $53 \%$ de mistura de stigmasterol, campesterol e brassicasterol.

O sangue para as medidas dos lípides plasmáticos foi colhido em tubos contendo $1 \%$ EDTA após $12 \mathrm{~h}$ de jejum, no $21^{\circ}$ e no $28^{\circ}$ dia de cada período de estudo, e centrifugado em baixa rotação para separação do plasma. Uma alíquota de plasma foi imediatamente submetida à precipitação de apo Bpara obtenção do HDL-C. O plasma total e o HDL-C foram estocados $\mathrm{a}-70^{\circ} \mathrm{C}$. Todas as medidas foram realizadas no final do estudo, em duplicata, para evitar a influência de variações inter-ensaios, com uso de métodos enzimáticos comercialmente disponíveis. O LDL-C foi obtido pela fórmula de Friedwald. Foi realizada extração de DNA para análise de apo Eatravés do método descrito por Miller S.A ${ }^{18,19}$.

Os resultados obtidos foram analisados segundo testes estatísticos adequados, com nível de significância de $\mathrm{P}<0,05$.

\section{Resultados}

A tabela I apresenta os valores, em jejum, de lípides e de lipoproteínas plasmáticos medidos nos períodos basal, placebo e de margarina enriquecida com FE. As concentra- 


\begin{tabular}{|c|c|c|c|}
\hline \multicolumn{4}{|c|}{$\begin{array}{c}\text { Tabela I - Concentração plasmática }(\mathrm{mg} / \mathrm{dL}) \text { de colesterol, } \\
\text { triglicérides, HDL-colesterol e LDL-colesterol, nos períodos basal, } \\
\text { placebo e FE (média } \pm \text { DP). }\end{array}$} \\
\hline & Basal & Placebo & $\mathrm{FE}$ \\
\hline Colesterol & $269 \pm 31$ & $258 \pm 30$ & $243 \pm 31^{1,2}$ \\
\hline Triglicérides & $174 \pm 67$ & $162 \pm 60$ & $156 \pm 63$ \\
\hline HDL-C & $43 \pm 13$ & $44 \pm 12$ & $42 \pm 14$ \\
\hline LDL-C & $192 \pm 27$ & $182 \pm 27$ & $169 \pm 25^{3,4}$ \\
\hline \multicolumn{4}{|c|}{$\begin{array}{l}\text { Diferenças significativas pelo teste de ANOVA. Comparação com pós- } \\
\text { teste de Newman-Keuls: } 1 \text {. FE } \times \text { Basal }=p<0,001 ; 2 \text {. FE } \times \text { Placebo }= \\
p<0,01 ; 3 \text {. FE } \times \text { Basal }=p<0,001 ; 4 \text {. FE } \times \text { Placebo }=p<0,05 .\end{array}$} \\
\hline
\end{tabular}

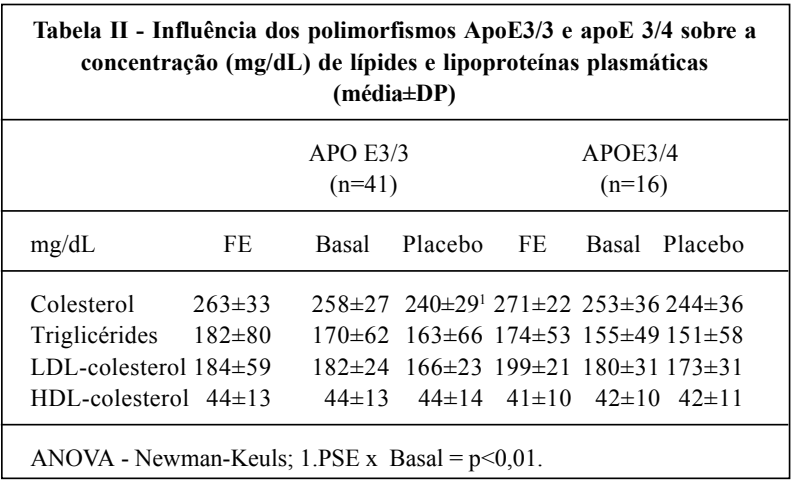

ções de colesterol total e de LDL-C diminuíram significativamente após quatro semanas do uso de fitoesteróis em comparação com o período placebo e basal. As concentrações de colesterol e de LDL-C diminuíram 10\% e 12\%,respectivamente, quando comparados ao período basal e $6 \%$ e $8 \%$, respectivamente, quando comparados ao placebo. Não foram observadas alterações nas concentrações plasmáticas de HDL-C e de triglicérides.

Não houve alteração na resposta da colesterolemia e do LDL-C comparando-se os dois diferentes fenótipos estudados, E3/3 eE3/4. Foi encontrada diferença apenas comparando-se o grupo $\mathrm{PSE}$ ao Basal nos pacientes E3/3.

A redução do LDL-C com fitoesteróis, em valores absolutos $(\mathrm{mg} / \mathrm{dL})$ variou de acordo com os valores basais de LDL-C, sendo mais intensa quanto maior foi o valor do LDL-C basal (fig. 1).

\section{Discussão}

Vários estudos, utilizando diferentes métodos experimentais, mostraram que fitoesteróis competem com o colesterol no momento da absorção intestinal, reduzindo, portanto, a concentração plasmática do colesterol ${ }^{1-4,20-22}$, o que pode ser também atribuído a um aumento da expressão do receptor da LDL no figado ${ }^{23}$. O presente trabalho confirma e estende as observações prévias, mostrando que o consumo de $20 \mathrm{~g} /$ dia de margarina enriquecida com fitoesterol diminuiu as concentrações plasmáticas de colesterol e LDL-colesterol, enquanto os triglicerídeos e o HDL-colesterol não se modificaram. Além do mais, os indivíduos com maiores

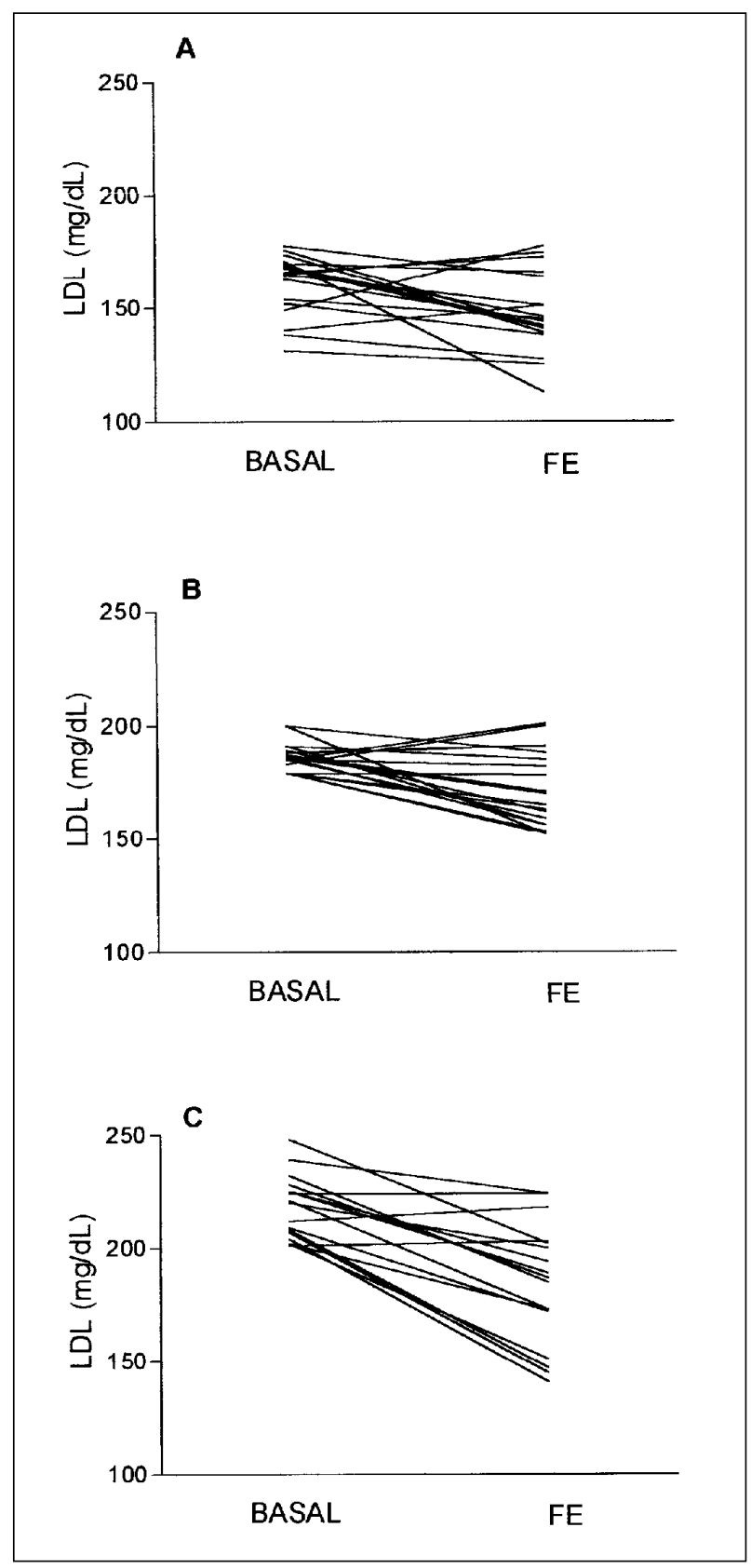

Fig. 1 - Reduções médias da concentração de LDL-colesterol (mg/dL) provocadas pelo $F E$ variando de acordo com o aumento do intervalo da concentração (entre parênteses), A) - 12,7mg/dL (131 a 178mg/dL, n=18); B) - 15,8 mg/dL (178 a 200mg/dL, $\mathrm{n}=20) ; \mathrm{C})-32,8 \mathrm{mg} / \mathrm{dL}, \mathrm{n}=20$ ). Analise de variância ANOVA, $\mathrm{p}=0,0054$. Comparação compós teste de Newman-Keuls: $\mathrm{Cx}$ A: $<<0,01 ; \mathrm{Cx}$ B: $\mathrm{p}<0,01 ; \mathrm{A}$ x B: não significante.

concentrações de LDL-colesterol, $>200 \mathrm{mg} / \mathrm{dL}$, foram os que mais se beneficiaram com o tratamento. O consumo moderado de margarina sem fitoesterol (placebo) não teve efeito desfavorável sobre os lípides plasmáticos.

$O$ fato de os indivíduos serem E3/3 ou E3/4 não influenciou a resposta da colesterolemia e do LDL-C, ao contrário do que se esperava, uma vez que os portadores de genótipo E4 são aqueles que absorvem maior quantidade de colesterol da dieta. Não foi encontrada diferença estatisticamente significativa, possivelmente em razão do diferente 
número de pacientes estudados nos dois grupos $(\mathrm{E} 3 / 3=41$ eE3/4=16).

Em resumo, este estudo conclui que os ésteres de fitoesteróis são capazes de reduzir a colesterolemia em nossa população, e que este objetivo é alcançado mesmo elevando-se a ingestão de margarina acima do hábito da população brasileira. Conclui, também, que se beneficiam mais aqueles que têm valores maiores de colesterol plasmático e que, na prática médica, são exatamente os que habitualmente se submetem a drogas redutoras da colesterolemia.

É necessário saber, no entanto, se a população brasileira apresentará, a longo prazo, boa aderência ao uso da margarina, uma vez que o consumo recomendado é maior do que o habitual. Deve-se lembrar também, que o produto não é recomendado para pacientes com beta-sitosterolemia, doença extremamente rara, na qual a absorção dos fitosteróis encontra-se aumentada ${ }^{24}$.

\section{Agradecimentos}

Ao Dr. Jõao Nóbrega (Cardio Center), ao Instituto dos LIMs do Hospital das Clínicas da FMUSP (São Paulo) e Faculdade de Medicina de Ribeirão Preto - USP pela seleção dos pacientes, ao suporte técnico de Patrícia M. Cazita e Vivian Buonacorso e à indústria Gessy Lever Ltda., SP.

\section{Referências}

1. Miettinen TA, Gylling H. Regulation of cholesterol metabolism by dietary plan sterols. Curr Opin Lipidol 1999; 10: 9-14.

2. Less AM, Mok HYI, Lees RS, McCluskey MA, Grundy SM. Plant sterols as cholesterol-lowering agents clinical trials in patients with hypercholesterolemia and studies of sterol balance. Atherosclerossis 1977; 28: 325-38.

3. Hallikainem MA, Uusitupa MJ. Effects of 2 low-fat stanol ester-containing margarines on serum cholesterol concentrations as part of a low-fat diet in hypercholesterolemic subjects. Am J Clin Nutr 1999: 69: 403-10.

4. Ostlund RE, Spilburg CA, Stenson WF. Sitostanol administered in lecithin micelles potently reduces cholesterol absorption in humans. Am J Clin Nutr 1999; 70: 826-31.

5. Law M. Plant sterol and stanol margarines and health. Br Med J 2000; 320: 861-4.

6. Andersson A, Karlström B, Mohsen R, Vessby B. Nome do artigo???? Eur Heart J Supplements 1999; 1(suppl S): S80-S90.

7. Wsetstrate JA, Meijer GW. Plant sterol-enriched margarines and recuction of plasma total-and LDL-cholesterol concentrations in normocholesterolaemic and mildly hypercholesterolaemic subjects. Eur J Clin Nutr 1998; 52: 334-43.

8. Miettinen TA, Puska P, Gylling H, Vanhanen H, Erkki V. Reduction os serum cholesterol with sitostanol-ester margarine in a mildy hypercholesterolemic population. N Engl J Med 1995; 333: 1308-12.

9. Tall A, Welch C, Applebaum-Bowden D, Wassef M. Interaction of diet and genes in atherosclerosis. Arterioscler Thromb Vasc Biol 1997; 17: 3326-31.

10. Willians $\mathrm{CM}$. Gene- nutrient interaction: an important area for considaration. $\mathrm{Br}$ J Nutr 1998; 79: 115.

11. Mahley RW, Ji ZS. Remnant lipoprotein metabolism: key pathways involving cell-surface heparan sulfate proteoglycans and apolipoprotein. J Lipid Res 1999; 40: 1-16.

12. Gylling H, Kontula K, Miettinen TA. Cholesterol absorption and metabolism and LDL kinetics in healthy men with different apoprotein E phenotypes and apolipoprotein B Xba I and LDL receptor Pvu II genotypes. Arterioscler Thromb Vasc Biol 1995; 15: 208-13.
13. Kuust GH, Vanhanen H, Mittinen TA. Apolipoprotein E phenotype and cholesterol metabolism in familial hypercholesterolemia. Atherosclerosis 1989; 80: 27-32.

14. Kesaniemi YA, Ehnholm C, Miettinen TA. Intestinal cholesterol absorption efficiency in man is related to apolipoprotein E phenotype. J Clin Invest 1987; 80: 578-81.

15. Miettinen T, Vanhanen H. Dietary sitostanol related to absorption, synthesis and serum level of cholesterol in different apolipoprotein E phenotypes. Atherosclerosis 1994; 105: 217-26.

16. Vanhanen HT, Blomqvist S, Ehnholm C, et al. Serum cholesterol, cholesterol precursors and plant sterols in hypercholesterolemic subjects with different apoE phenotypes during dietary ' sitostanol ester treatment. J Lipid Res 1993; 34: 1535-43.

17. Lichtenstein AH, Deckelbaum RJ. Stanol/sterol ester-containing foods and blood cholesterol levels. Circulation 2001; 103: 1177-9.

18. Miller SA, Dykes DD, Polesky HF. A simple salting out procedure for extracting DNA from human nucleated cells. Nucleic Acid Res 1988; $16: 1215$.

19. Hixson JE, Vernier DT. Restriction isotyping of human apolipoprotein E by gene amplification and cleavage with Hha I. J Lipid Res 1990; 31: 545-8.

20. Wester I. Cholesterol-lowering effect of plant sterols. Eur J Lipid Sci Technol 2000; 37-44.

21. Pollak OJ. Reduction of blood cholesterol in man. Circulation 1953; 7: 702-6.

22. Armstrong MJ, Carey MC. Thermodynamic and molecular determinants of sterol solubilities in bile salt micelles. J Lipid Res 1987; 28: 1144-55.

23. Plat J, Mensink RP. Consumption of plant stanol esters increase LDL receptor expression in mononuclear cels from non-hipercholesterolemic subjects Atherosclerosis 2000; $151: 86$

24. Salen G., Shefer S, Nguyen L, Ness GC, Tint GS, Shore V. Sitosterolemia J Lipid Res 1992; 33: 945-54. 